\title{
Effect of Vacancies on Electronic and Magnetic Properties of Hydrogen Passivated Graphene Nanoribbons
}

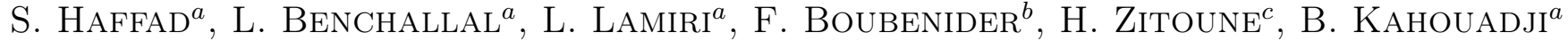 \\ AND M. SAMAH ${ }^{a, *}$ \\ ${ }^{a}$ Université A/Mira, Bejaia, Algeria \\ ${ }^{b}$ Université Houari Boumediene, Laboratoire de physique des matériaux, Alger, Algeria \\ ${ }^{c}$ Université Mohand Oulhadj, Bouira, Algeria \\ (Received December 20, 2017; in final form March 26, 2018)
}

\begin{abstract}
Using first-principles calculations we have demonstrated that electronic and magnetic properties of armchair graphene nanoribbons are modified by introducing vacancies defects. The equilibrium geometries, electronic, charge spin density distributions, electronic band structures, and magnetic moments were examined in the presence of vacancies. We have found that introducing vacancies into armchair graphene nanoribbons changes the spatial distribution of neighbor atoms, particularly those located around the vacancies. Our calculations showed that the vacancies have significant effect on the magnetization of armchair graphene nanoribbons. Magnetic moment values and electronic behavior in different configurations depend on the number of vacancies. These results suggest that vacancy defects can be used to modify the electronic and the magnetic properties of armchair graphene nanoribbons.
\end{abstract}

DOI: 10.12693/APhysPolA.133.1307

PACS/topics: DFT, vacancies, AGNR, magnetic, passivated, electronic, charge transfer, band structure

\section{Introduction}

The fascinating graphene nanoribbons (GNRs) are quasi-one-dimensional structures of carbon. These structures can be constructed as strips of graphene sheet, the two-dimensional honeycomb lattice of carbon with $s p^{2}$ hybridization. Geometrically two main types of GNRS can be can exist: zigzag edge and armchair edge [1, 2]. The edge geometry is the key parameter which determines the electronic properties of the nanoribbons [3-6]. Density functional calculations show that all GNRs are semiconductors with band gaps which depend on their width and edge geometry. For example, the energy gaps of the armchair GNRs depend on the number of dimer lines $N_{a}$ along the ribbon width. Armchair GNRs (AGNRs) with $N_{a}=15\left(N_{a}=3 p\right)$ and $N_{a}=16\left(N_{a}=3 p+1\right)$ display energy gaps $E_{g}=556 \mathrm{meV}$ and $E_{g}=657 \mathrm{meV}$ respectively, whereas the armchair GNR with $N_{a}=17$ $\left(N_{a}=3 p+2\right)$ is predicted to exhibit a much smaller gap $E_{g}=118 \mathrm{meV}[6-12]$.

Moreover, the electronic structure of graphene nanoribbons can be modified by chemical functionalization. A large variety of electronic and magnetic properties, such as semiconducting with a wide range of band gap, metallic, ferromagnetic, antiferromagnetic, halfmetallic, half-semiconducting, can be obtained by chemical modifications of the nanoribbons leading for different options of functionalizations of these materials [13-16].

From a practical point of view, when nanoribbons are fabricated they will have usually some structural defects. Vacancy defects are among the most probable

\footnotetext{
*corresponding author; e-mail: madanisamah@yahoo.fr
}

ones. These defects should be taken into account in practical aspects of the electronic properties in nanodevices based on graphene nanoribbons [17]. Elsewhere, it is well demonstrated that high electrons and ions radiations on GNRs induce structural defects such as vacancies showing an alternate ferromagnetic and antiferromagnetic behavior of the structure [17-22].

The study of the effects of defects on graphene nanoribbons is interesting because their band gap, magnetic state and symmetry are expected to be influenced. Calculations based on the mean-field Hubbard model by Palacios et al. [23], have revealed interesting electronic and magnetic properties of single vacancy and voids in armchair nanoribbons. Topsakal et al. [24] have demonstrated that the band-gap of GNR changes depend on the width of the ribbon as well as on the position of the void relative to the edges of the ribbon. Repeating vacancy or divacancies in GNR induce metallization as well as magnetization in nonmagnetic semiconducting nanoribbons due to the spin polarization of local defect states. Antiferromagnetic ground state of semiconducting zigzag ribbons can change to ferrimagnetic state upon creation of vacancy defects. Experimental results obtained by Meyer et al. shows clearly a vacancy defect on GNR. The formation of these defects is observed in situ [25].

In this article we will study theoretically the effects of vacancies defects on electronic and magnetic properties of graphene nanoribbons using DFT implemented in SIESTA code.

\section{Computational methods}

The atomic-scale reconstructions are carried out by using the density functional theory implemented within the SIESTA simulation package $[26,27]$. Standard normconserving Troullier-Martins pseudopotential is utilized 
to describe the interaction between valence electrons and the atomic core [28]. The graphene nanoribbon is inscribed in the $y z$ plane forming a rectangle of sides of $16.68 \AA$ and $15.62 \AA$. The supercell containing this nanoribbon has the following dimensions: $25 \AA$ in $x$ direction, $25 \AA$ in $z$ direction and 17.04 in $y$ direction. GNR is composed by 120 atoms; 104 carbon atoms and 16 hydrogen ones. The generalized gradient approximation (GGA) in the framework of functional proposed by Perdew, Burke, and Ernzerhof (PBE) was adopted for exchange and correlation [29]. Kinetic energy cutoff was $400 \mathrm{Ry}$, and double- $\zeta$ plus polarization (DZP) basis set is chosen in the numerical calculation. All nanostructure geometries were relaxed until atomic forces were below
$0.01 \mathrm{eV} / \AA$. In all our relaxation calculus of AGNR structure, the Brillouin zone (BZ) is sampled by $1 \times 16 \times 1$ special $k$ points and the interatomic forces were smaller than $0.01 \mathrm{eV} / \AA$ and the energy of convergence between two steps were chosen equal to $10^{-5}$. The conjugate gradient method is used to optimize all our AGNR structures.

\section{Results and discussion}

\subsection{Structural relaxation}

Figure 1 shows the fully relaxed atomic structures of pristine and defected GNRs with 1, 2,3,4,5,6, 12 and 16 vacancies (voids) where carbon atoms are removed.
123 .1213

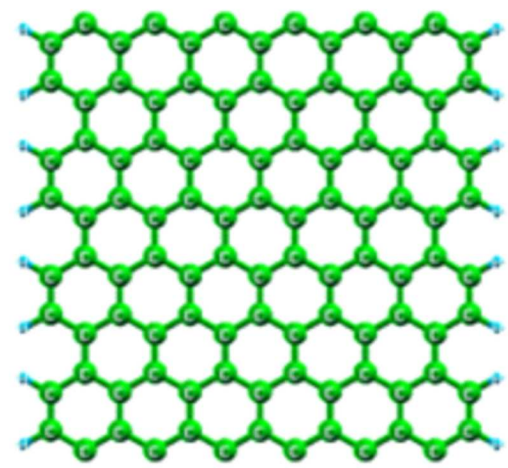

$-\mathrm{A}-$

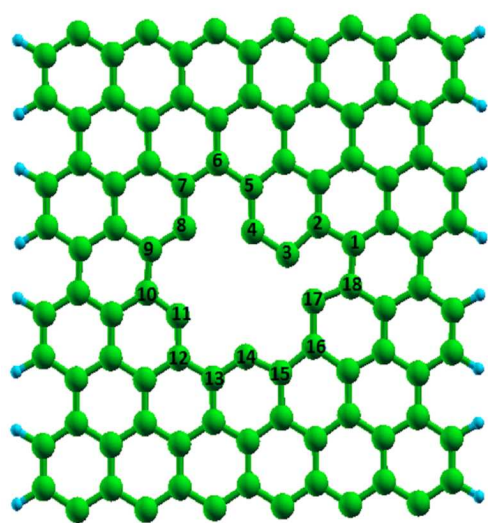

-D-

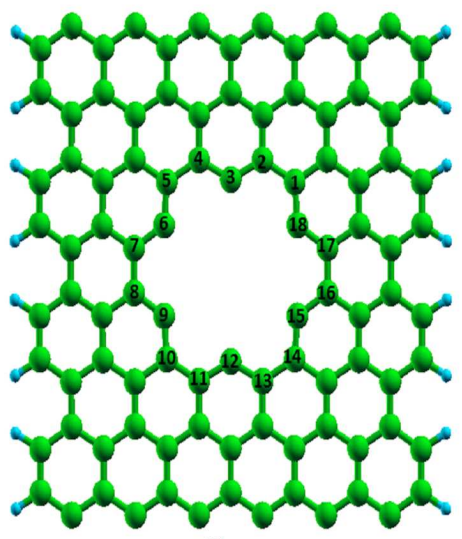

-G-

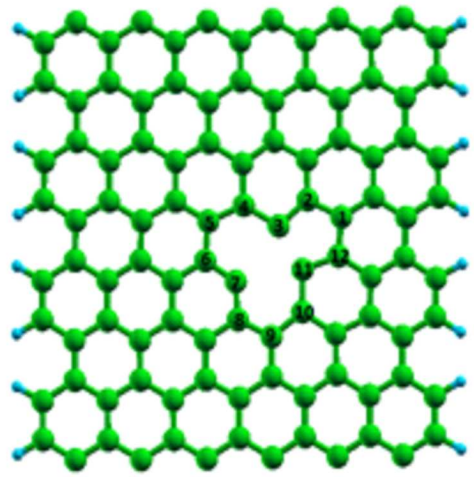

-B-

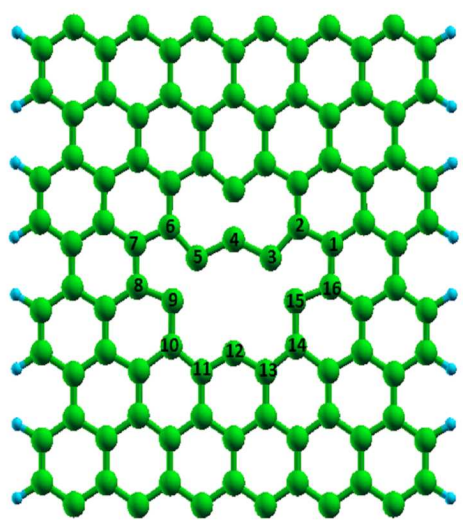

-E-

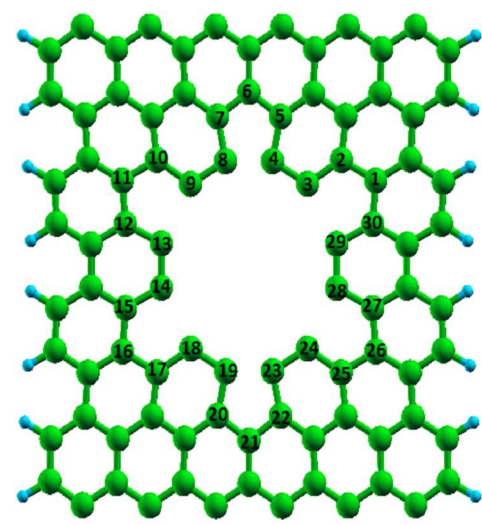

-H-

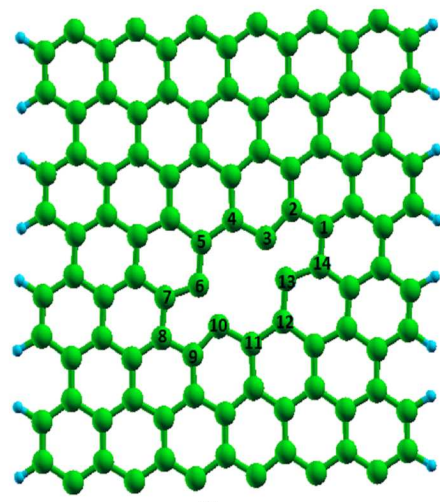

$-\mathrm{C}-$

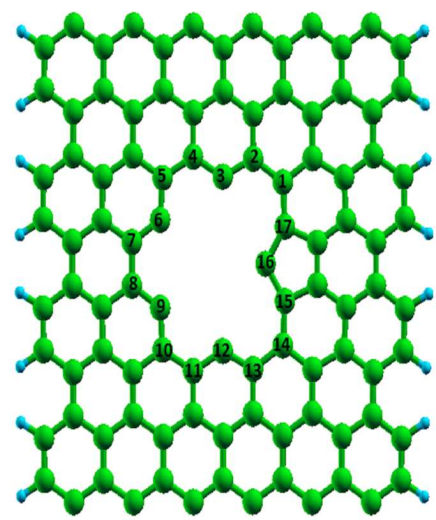

$-\mathrm{F}$ -

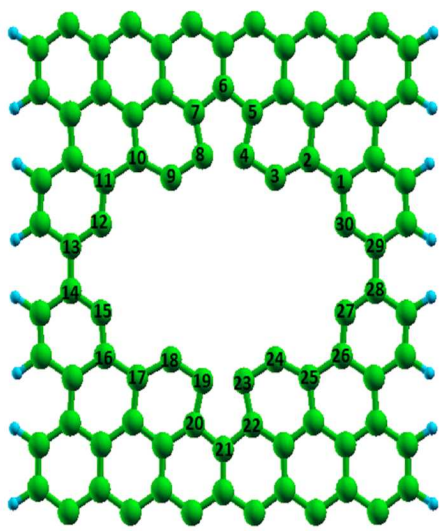

-I-

Fig. 1. Relaxed structures of A-pristine AGNR and B, C, D, E, F, G, H and I AGNRs with 1, 2, 3, 4, 5, 6, 12 and 16 vacancies. 
The relaxed pristine GNR (Fig. 1A) is constituted as a honeycomb structure with $\mathrm{C}-\mathrm{C}$ distance equal to $1.43 \AA$. Figure $1 \mathrm{~B}$ shows the fully relaxed one vacancy-GNR. It exhibits extended $\mathrm{C}-\mathrm{C}$ bond lengths in comparison with those of standard pristine GNR. It is important to note that in our calculation no Jahn-Teller distortion was observed. All carbon atoms are described in the plane of the structure. Two of the three dangling bonds are connected to each other and towards the missing atom. We have to note that such as effect has been already obtained by earlier studies. While some have reported planner structure [22] others have found non-planar structures with different out-of-plane displacements [30-32]. The three dangling bonds surrounding the vacancy tend to form one reconstructed bond with length of $2.07 \AA$ that has to be compared to the 2 nd neighbors distance of $2.46 \AA$ in pristine honeycomb. The formation energy of the V1(5-9)defect is quite high. Ab initio calculations provide a value of $8 \mathrm{eV}$ similar to that obtained by El-Barbary et al. [30].

Based on single vacancy defects, if there is another loss of a carbon atom, it will be a divacancy defect. The divacancy -GNR, as shown in Fig. 1C exhibits a $5-8-5[33,34]$ scheme which possesses a $D_{2 h}$ symmetry since two pentagons and one octagon bond appear instead of four hexagons in perfect GNRs. The 5-8-5 defects makes the GNRs remarkably shrink toward the center of the octagon and correspondingly, the four hexagons that connected with the octagon have been twisted by a certain angle. The $\mathrm{C}-\mathrm{C}$ length is approximately reduced to $4.05 \AA$ compared with the pristine $\mathrm{C}-\mathrm{C}$ bond length $(4.86 \AA)$. A similar results are obtained by Tarawneh et al. [32]. It is found that the length of the newly formed $\mathrm{C}-\mathrm{C}$ bond substantially stretches to $1.64 \AA$ and the other $\mathrm{C}-\mathrm{C}$ bonds that form the octagon. Comparable results are obtained by Zhao et al. [35]. The defect has formation energy of about $8 \mathrm{eV}$, which is of the same order as a single vacancy.

One step further would be the transformation of the second vacancy defect into more complicated vacancy defects by the removal of more carbon atoms resulting in larger and more complex defect reconstructions. The next targeted defected GNR is the three vacancies, noted below as 3 -v-GNR created by missing three carbon atoms. Figure $1 \mathrm{D}$ shows the relaxed $3-\mathrm{v}-\mathrm{GNR}$. A $\mathrm{C}-\mathrm{C}$ distance around the voids produced by the vacancies seems to be shorter than in pristine GNR. For example, the bond length of $\mathrm{C}_{12}-\mathrm{C}_{4}$ bound equal to $3.71 \AA$ where it is about $4.23 \AA$ in the pristine GNR. Figure $1 \mathrm{E}$ to $1 \mathrm{I}$ represent the relaxed configurations of four to sixteen vacancies in GNRs. Same remarks as done for first structures can be mentioned.

Figure 2 shows the variation of formation energy per atom versus number of vacancies. A linear variation can be seen easily. More is the number of vacancies, greater is the instability of the structure. Vacancy formation is an endothermic process. Consequently, these defected configurations tend to minimize their en-

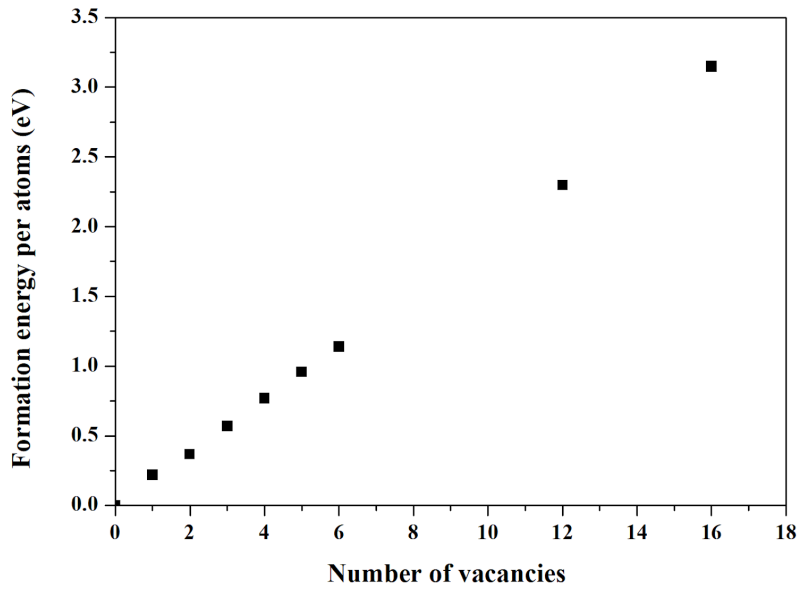

Fig. 2. Variation of formation energy per atoms versus number of vacancies.

ergy by absorbing or adsorbing atoms or molecules. This effect will be studied in our future articles.

Vacancy concentration of $9 \%$ is made possible for graphene synthesized through the chemical reduction of graphene oxide [36] and an even higher vacancy concentration might be possible for graphene oxide with $30 \%$ mass loss after thermal exfoliation [37]. In addition, accumulation of irradiation-induced damage using molecular dynamics simulation reveal that graphene membrane with vacancy concentrations of at least $35 \%$ does not show any signs of structural failure [38].

Figure $1 \mathrm{G}, \mathrm{H}$ and I exhibits what we commonly named flower defects. Flower defect is a nonlinear topological defect consisting of centro-symmetric forms. According to study of Ref. [39], flower defects are energetically more favorable. When graphene is produced at high temperatures, some of the graphene sections cut loose [39]. When cooled, these sections rotate and patch up on the graphene lattice in a non-linear manner like a flower. Hence this defect is named as flower defect. For the 6vacancies (6V) GNR, the void produced is very symmetric. Such a results are obtained by Islam et al [40].

\subsection{Electronic and magnetic properties}

Vacancies in graphene have been proposed to give rise to like magnetism in carbon materials, a conjecture which has been supported by recent experimental evidence. The main element in this "vacancy magnetism" is the formation of magnetic moments in vacancy-induced electronic states [41].

Firstable, the band structure of perfect 13-AGNR was calculated and plotted in Fig. 3. As shown, this nanoribbon is a semiconductor with a band gap of about $0.96 \mathrm{eV}$. Table I shows that the pristine nanoribbon exhibits a nonmagnetic (NM) behavior with a zero magnetic moment. Son et al. [42] show that all armchair GNRs are semiconductors with energy gaps which decrease as a function of increasing ribbon widths. No magnetism has 
been found in armchair GNRs. We easily remark that there are four important subbands which dominate the electronic behaviors of the GNR. These subbands are constituted by the $\pi$ bonds of carbon atoms, and have different shapes. These results are corroborated by those obtained by Sun et al. [43]. It can be seen also that op- posite spin polarization bands of the pristine AGNR are completely degenerate. The highest occupied band A and the lowest unoccupied band A' move away from the Fermi level at the $\Gamma$ point, opening a symmetric band gap, thereby the band structure of the pristine AGNR manifests semiconductor properties [44].
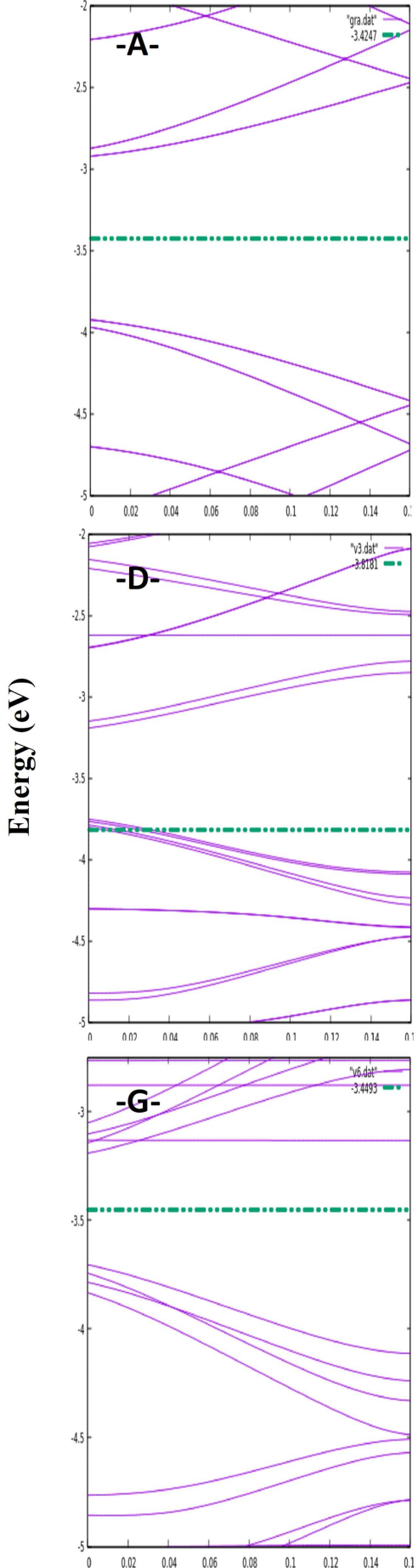
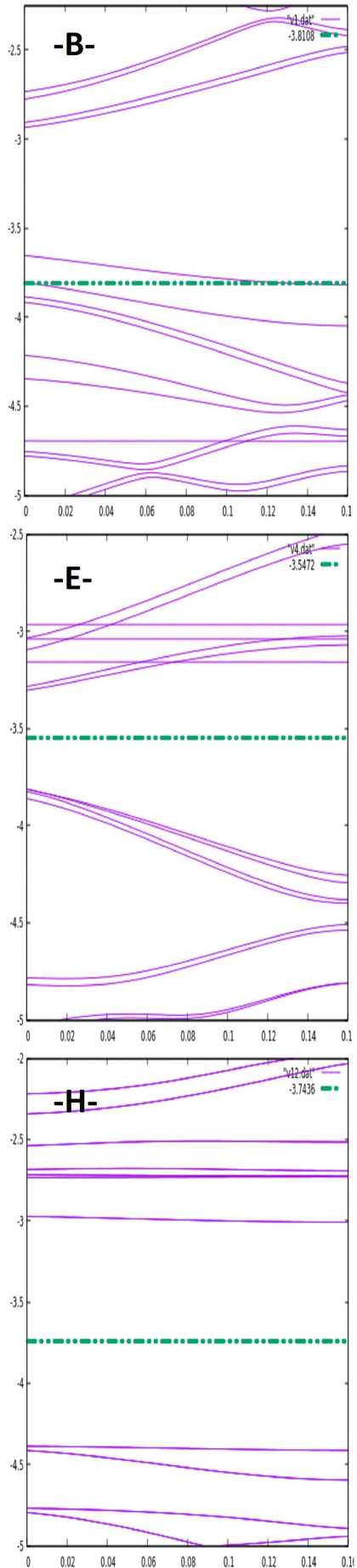
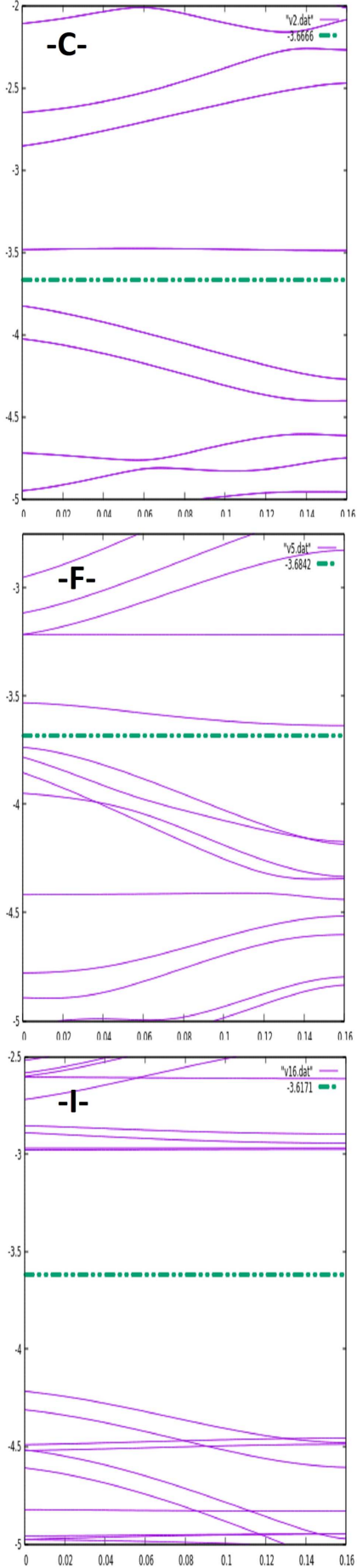

\section{$k$ (wave vector)}

Fig. 3. Bands structures of A-pristine AGNR and B, C, D, E, F, G, H and I AGNR with 1, 2, 3, 4, 5, 6, 12 and 16 vacancies (Fermi level is drawn with dashed line). 
TABLE I

Gap energies, magnetic moment and nature of AGNRs with different number of vacancies.

\begin{tabular}{c|c|c|c}
\hline $\begin{array}{c}\text { Number } \\
\text { of vacancies }\end{array}$ & $E_{g}[\mathrm{eV}]$ & $M\left(\mu_{\mathrm{B}}\right)$ & $\begin{array}{c}\text { Nature } \\
\text { of material }\end{array}$ \\
\hline 0 & 0.98 & 0 & $\mathrm{Sc}$ \\
1 & 0.0 & 1.65 & metal \\
2 & 0.33 & 0 & $\mathrm{Sc}$ \\
3 & 0.0 & 1.00 & metal \\
4 & 0.46 & 2.00 & $\mathrm{Sc}$ \\
5 & 0.10 & 5.93 & $\mathrm{Sc}$ \\
6 & 0.47 & 6.00 & $\mathrm{Sc}$ \\
12 & 1.36 & 0 & $\mathrm{Sc}$ \\
16 & 1.23 & 8.00 & $\mathrm{Sc}$
\end{tabular}

After introducing a single vacancy, the NM ground state changes to a ferromagnetic (FM) state and the resulting magnetic moment of the super cell is equal to $1.65 \mu_{\mathrm{B}}$. These results are in excellent agreement with those obtained by Longo et al. [45]. This value is relatively small compared with other defected GNRs because of the relative relaxation of carbon atoms near the position of the vacancy and the interaction with the edge. Carbon atom numbered 7 in Fig. 1 contributes to the major part for the magnetic moment. Since the vacancy site is located at the center of the nanoribbon, the spin around the vacancy is strongly distributed and the semiconducting state changes to a metal-like state (see Fig. 3B). More recently, Safari et al. [46], using the VASP code, have obtained a magnetic moment of about $0.79 \mu_{\mathrm{B}}$ for an armchair graphene nanoribbons. These differences are due to different used codes and the size of the AGNR.
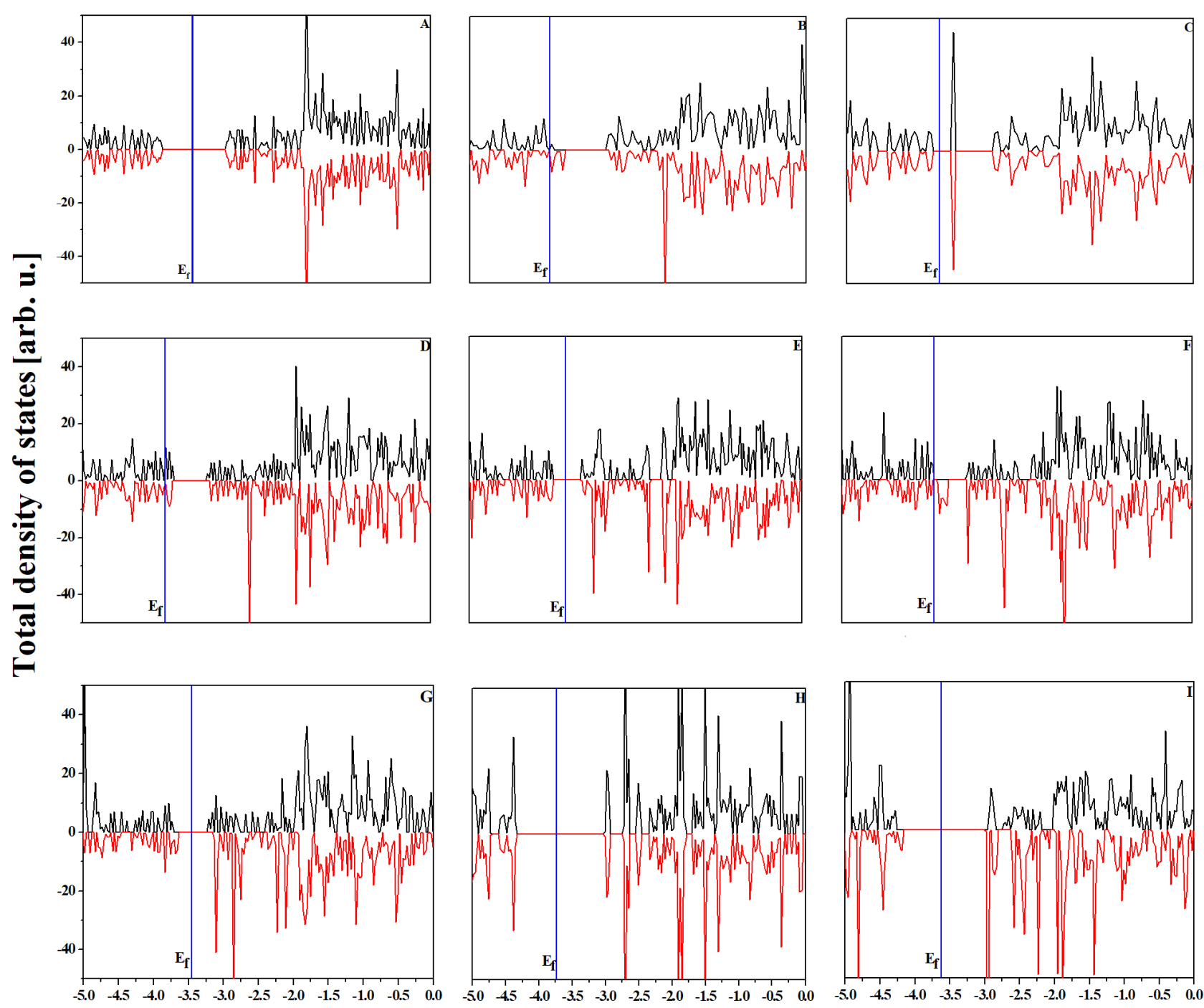

Fig. 4. Total density of states of A-pristine AGNR and defected AGNRs (B, C, D, E, F, G, H and I) with 1, 2, 3, 4, 5, 6, 12 and 16 vacancies (black line are spin-up contribution and red line are spin-down one). 
GNR with divacancy has a net zero magnetic moment when two carbon atoms are removed from different sublattices (A and $\mathrm{B})$. This is due to the antiferromagnetic coupling of $\mathrm{A}-\mathrm{B}$ sublattices. The same results are obtained by Tarawneh et al. studying 6 -ZGNRs [32]. The 5-8-5 defect creates a defect state labeled $g$ that appears slightly above the Fermi level. This $g$-state hybridizes with the A-A'-bands to evolve into the B and B'-bands. The defect subband induced by the asymmetric 5-8-5 defect, denoted by $\gamma$ does not cross the Fermi level, in good agreement with results of Zhao et al. [47]. Correspondingly, the dispersion between its highest occupied B-band and the lowest unoccupied B'-band at the $\Gamma$-point is substantially narrowed.

For 3-vacancies defected GNR, the electronic bands structure is depicted in Fig. 3D. The calculated band gap is about $0.52 \mathrm{eV}$. The three unoccupied subbands lie very closely with the Fermi level. The estimated magnetic moment is about $1 \mu_{\mathrm{B}}$. The most major part on this magnetic moment come from the contribution of the atom labeled 12 in Fig. 1. This contribution is about $0.9 \mu_{\mathrm{B}}$.

$4-\mathrm{V}$, and $6-\mathrm{V}$, respectively GNRs with four and six vacancies, exhibit a medium energy gaps $(0.45$ and $0.54 \mathrm{eV}$, respectively). Their magnetic moments equal to 2 and $6 \mu_{\mathrm{B}} .4-\mathrm{V}$ and $6-\mathrm{V}$ have the greatest magnetic moments because of the highest number of dangling bonds. Elsewhere, Jokar and Moslemi [48], studying the armchair 11-GNR, have obtained that 4-vacancies triangle-type remains metallic either the position of the void. They obtain also that $6-\mathrm{V}$ GNR is always semiconductor with gap between 0.09 to $0.89 \mathrm{eV}$ depending on the position of the void. These differences can be explained by the width of ribbons around the vacancy and vacancy location. Topsakal et al. [26], using the VASP package, have demonstrated that the 6 -V-GNR properties depend on the width of nanoribbons and the position of the void. In addition, states localized around the defect form flat bands near the edge of valence and conduction bands because of their reduced coupling. These results are in agreement with previous works revealing states localized at the vacancy defect [49-51]. The 5-v GNR exhibits an indirect gap and magnetic moment of about $5.93 \mu_{\mathrm{B}} .12-$ $\mathrm{v}$ and $16-\mathrm{v}$ GNRs are semiconductors with approximately the same gaps, equal to $1.41 \mathrm{eV}$ and magnetic moments about 0 and $8 \mu_{\mathrm{B}}$, respectively. From Fig. 4, we see that $12-\mathrm{v}$ and $16-\mathrm{v}$ GNRs are semiconductors with approximately the same gaps, equal to $1.41 \mathrm{eV}$ and magnetic moments about 0 and $8 \mu_{\mathrm{B}}$ respectively.

\section{Conclusion}

The first-principles pseudopotential method combined with the generalized gradient approximation has been employed to study the structural, electronic and magnetic properties of AGNRs with vacancies. We optimized the equilibrium structures of 13-AGNR with a several vacancies complexes. It was found that introducing vacancies into the AGNR changes the arrangement of the atoms that are located around the vacancies. Our calculations demonstrated that vacancies can impose a significant effect on the magnetization and electronic structure of graphene nanoribbons.

\section{References}

[1] Y. Niimi, T. Matsui, H. Kambara, K. Tagami, M. Tsukada, H. Fukuyama, Appl. Surf. Sci. 241, 43 (2005).

[2] Y. Kobayashi, K.-I. Fukui, T. Enoki, K. Kusakabe, Y. Kaburagi, Phys. Rev. B 71, 193406 (2005).

[3] D.J. Klein, Chem. Phys. Lett. 217, 261 (1994).

[4] M. Fujita, K. Wakabayashi, K. Nakada, K. Kusakabe, J. Phys. Soc. Jpn. 65, 1920 (1996).

[5] R. Saito, M. Fujita, G. Dresselhaus, M.S. Dresselhaus, Appl. Phys. Lett. 60, 2204 (1992).

[6] Y.-W. Son, M.L. Cohen, S.G. Louie, Phys. Rev. Lett. 97, 216803 (2006).

[7] K. Nakada, M. Fujita, G. Dresselhaus, M.S. Dresselhaus, Phys. Rev. B 54, 17954 (1996).

[8] Y.-W. Son, M.L. Cohen, S.G. Louie, Nature 444, 347 (2006).

[9] V. Barone, O. Hod, G.E. Scuseria, Nano Lett. 6, 2748 (2006).

[10] L. Yang, C.-H. Park, Y.-W. Son, M.L. Cohen, S.G. Louie, Phys. Rev. Lett. 99, 186801 (2007).

[11] O.V. Yazyev, Acc. Chem. Res. 46, 2319 (2013).

[12] W.-X. Wang, M. Zhou, X. Li, S.-Y. Li, X. Wu, W. Duan, L. He, Phys. Rev. B 93, 241403 (2016).

[13] M. Maruyama, K. Kusakabe, S. Tsuneyuki, K. Akagi, Y. Yoshimoto, J. Yamauchi, J. Phys. Chem. Solids 65, 119 (2004).

[14] D. Gunlycke, J. Li, J.W. Mintmire, C.T. White, Appl. Phys. Lett. 91, 112108 (2007).

[15] O. Hod, V. Barone, J.E. Peralta, G.E. Suseria, Nano Lett. 7, 2295 (2007).

[16] N. Gorjizadeh, A.A. Farajian, K. Esfarjani, Y. Kawazoe, Phys. Rev. B 78, 155427 (2008).

[17] A. Hashimoto, K. Suenaga, A. Gloter, K. Urita, S. Iijima, Nature 430, 870 (2004).

[18] K. Nordlund, J. Keinonen, T. Mattila, Phys. Rev. Lett. 77, 699 (1996).

[19] P. Esquinazi, D. Spemann, R. Höhne, A. Setzer, K.H. Han, T. Butz, Phys. Rev. Lett. 91, 227201 (2003).

[20] O.V. Yazyev, L. Helm, Phys. Rev. B 75, 125408 (2007).

[21] L. Brey, H.A. Fertig, S. Das Sarma, Phys. Rev. Lett. 99, 116802 (2007).

[22] M.A.H. Vozmediano, M.P. Lopez-Sancho, T. Stauber, F. Guinea, Phys. Rev. B 72, 155121 (2005).

[23] J.J. Palacios, J. Fernández-Rossier, L. Brey, Phys. Rev. B 77, 195428 (2008).

[24] M. Topsakal, E. Aktürk, H. Sevinçli, S. Ciraci, Phys. Rev. B 78, 235435 (2008).

[25] J.C. Meyer, C. Kisielowski, R. Erni, M.D. Rössell, M.F. Crommie, A. Zettl, Nano Lett. 8, 3582 (2008).

[26] P. Ordejón, E. Artacho, J.M. Soler, Phys. Rev. B 53, 10441 (1996). 
[27] J.M. Soler, E. Artacho, J.D. Gale, A. García, J. Junquera, P. Ordejón, D. Sánchez Portal, J. Phys. Condens. Matter. 14, 2745 (2002).

[28] N. Troullier, J. Luriaas Martins, Phys. Rev. B 43, 1993 (1991).

[29] J.P. Perdew, K. Burke, M. Ernzerhof, Phys. Rev. Lett. 77, 3865 (1996).

[30] A.A. El-Barbary, R.H. Telling, C.P. Ewels, M.I. Heggie, P.R. Briddon, Phys. Rev. B 68, 144107 (2003).

[31] Y. Ma, P.O. Lehtinen, A.S. Foster, R.M. Nieminen, New J. Phys. 6, 68 (2004).

[32] K. Tarawneh, N. Al-Aqtash, J. Nano. Res. 27, 65 (2014).

[33] F. Banhart, J. Kotakoski, A.V. Krasheninnikov, ACS Nano 5, 26 (2011).

[34] P.T. Araujo, M. Terrones, M.S. Dresselhaus, Mater. Today 15, 98 (2012).

[35] J. Zhao, H. Zeng, J. Wei, Physica B 407, 204 (2012).

[36] W. Gao, L.B. Alemany, L. Ci, P.M. Ajayan, Nat. Chem. 403, 8 (2009).

[37] H.C. Schniepp, J. Li, M.J. McAllister, H. Sai, M.H. Alonso, D.H. Adamson, R.K. Prud'homme, R. Car, D.A. Saville, I.A. Aksay, J. Phys. Chem. B 110, 8535 (2006).

[38] E.H. Åhlgren, J. Kotakoski, O. Lehtinen, A.V. Krasheninnikov, Appl. Phys. Lett. 100, 233108 (2012)
[39] E. Cockayne, G. Rutter, N. Guisinger, J. Crain P. First, J. Stroscio, Phys. Rev. B 83, 195425 (2011).

[40] M.S. Islam, S. Tanaka, A. Hashimoto, Carbon 80, 146 (2014).

[41] V.G. Miranda, L.G.G.V. Dias da Silva, C.H. Lewenkopf, Phys. Rev. B 94, 075114 (2016)

[42] Y.-W. Son, M.L. Cohen, S.G. Louie, Phys. Rev. Lett. 97, 216803 (2006).

[43] L. Sun, Q. Li, H. Ren, H. Su, Q. Shi, J. Yang, J. Chem. Phys. 129, 074704 (2008).

[44] H. Terrones, R. Lv, M. Terrones, M.S. Dresselhaus, Rep. Prog. Phys. 75, 062501 (2012).

[45] R.C. Longo, J. Carrete, L.J. Gallego, J. Chem. Phys. 134, 024704 (2011).

[46] E. Keshavarz Safari, A.A. Shokri, M. Babaei Pour, J. Magn. Magn. Mater. 441, 230 (2017).

[47] J. Zhao, H. Zeng, J. Wei, B. Li, D. Xu, Phys. Lett. A 378, 416 (2014).

[48] Z. Jokar, M.R. Moslemi, Int. J. Elect. Comp. Ener. Elect. Com. Eng. 9, 162 (2015)

[49] K. Wakabayashi, J. Phys. Soc. Jpn. 71, 2500 (2002).

[50] M.A.H. Vozmediano, M.P. Lopez-Sancho, T. Stauber, F. Guinea, Phys. Rev. B 72, 155121 (2005).

[51] M. Vitor, F. Pereira, J.M. Guinea, B. Lopes dos Santos, N.M.R. Peres, A.H. Castro Neto, Phys. Rev. Lett. 96, 036801 (2006). 